Pacific Journal of Mathematics

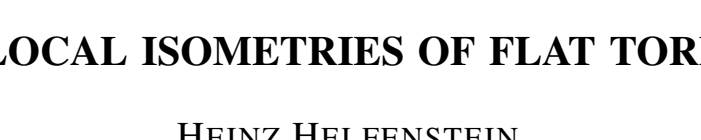




\section{LOCAL ISOMETRIES OF FLAT TORI}

\section{H. G. Helfenstein}

Let $T_{1}$ and $T_{2}$ be two flat tori (i.e., provided with a complete Riemannian metric of vanishing curvature). Since they are locally Euclidean each pair of points $P_{1}, P_{2}, P_{i} \in T_{i}$, has isometric neighborhoods. In general it is not possible, however, to join these separate isometries of neighborhoods to produce a single isometry $T_{1} \rightarrow T_{2}$ or $T_{2} \rightarrow T_{1}$; indeed there may not even exist a locally isometric map (of the whole surfaces). Necessary and sufficient conditions for the existence of such maps are deduced, making use of a recent conformal classification of maps between tori. As expected "ample" and nonample tori behave differently, and the determination of all local isometries leads to number-theoretic problems. Finally, for two given tori, the local isometries are compared with respect to homotopy by analyzing their effect on the fundamental groups.

Let $\boldsymbol{R}^{+}$denote the positive reals, $H$ the upper $z$-half-plane, and $S L(2, Z)$ the group of all $2 \times 2$ unimodular matrices with integral entries acting in the usual way as hyperbolic motions on $H$. The set of isometry classes of complete flat tori is parametrized by the 3dimensional manifold $\boldsymbol{R}^{+} \times(H / S L(2, Z))$. A point $\left(r^{2}, \tau\right)$ of this space represents the isometry class of the torus $E^{2} / \Gamma$, where $\Gamma$ is the group of Euclidean motions generated by the translations

$$
t_{1}(z)=z+r \quad \text { and } \quad t_{2}(z)=z+r h,
$$

with $h \in \tau$, (cf. [2]). Instead of "an isometry class of tori" we speak simply of "a torus". A torus $T=\left(r^{2}, \tau\right)$ is called ample if there exists $h \in \tau$ such that both $\Re h$ and $|h|^{2}$ are rational.

2. Riemannian covering maps. The following statements are generalizations of results obtained in [1] which can be similarly proved.

(i) For two tori $T_{i}=\left(r_{i}^{2}, \tau_{i}\right)$ there exist conformal covering maps $T_{1} \rightarrow T_{2}$ if and only if two representatives $h_{i} \in \tau_{i}$ are equivalent under the action of the group $G L^{+}(2, Q)=$ group of $2 \times 2$ matrices with rational entries and positive determinant.

(ii) Lifting any conformal covering $T_{1} \rightarrow T_{2}$ to the universal covering planes we obtain

$$
F(z, C, D)=C z+D,
$$

with complex constants $C \neq 0$ and $D$.

(iii) For nonample $T_{i}$ only 


$$
C(\kappa)=\frac{r_{2}}{r_{1}} \kappa, \quad \kappa= \pm 1, \pm 2, \cdots
$$

are admissible values in (1).

(iv) For ample $T_{i}=\left(r_{i}^{2}, \tau_{i}\right)$ (2) is replaced by

$$
C\left(\kappa_{1}, \kappa_{2}\right)=\frac{r_{2}}{r_{1}}\left(\kappa_{1}+\kappa_{2} q^{\prime \prime} s^{\prime \prime} h_{2}\right),
$$

where $h_{2} \in \tau_{2}, h_{1}=a h_{2}, a$ an integer, $\left(\kappa_{1}, \kappa_{2}\right) \neq(0,0)$ is a pair of arbitrary integers, and the integers $q^{\prime \prime}, s^{\prime \prime}$ are determined via the following relations,

$$
2 \Re h_{2}=\frac{p}{q}, \quad\left|h_{2}\right|^{2}=\frac{r}{s},
$$

$p, q>0, r>0, s>0$ integers,

$$
\begin{aligned}
& \text { g.c.d. }(p, q)=\text { g.c.d. }(r, s)=1, \\
g= & \text { g.c.d. }(q, s), q^{\prime}=q / g, s^{\prime}=s / g, \\
g^{\prime}= & \text { g.c.d. }(a, q), a^{\prime}=a / g^{\prime}, q^{\prime \prime}=q / g^{\prime}, \\
g^{\prime \prime}= & \text { g.c.d. }\left(a^{\prime}, s^{\prime}\right), a^{\prime \prime}=a^{\prime} / g^{\prime \prime}, s^{\prime \prime}=s^{\prime} / g^{\prime \prime} .
\end{aligned}
$$

The following materices are computable from these numbers.

$$
\widetilde{T}_{1}=\left(\begin{array}{ll}
a, & 0 \\
0, & 1
\end{array}\right), \quad \widetilde{T}_{2}=\left(\begin{array}{cc}
a^{\prime} p s^{\prime \prime}, & -a^{\prime \prime} q^{\prime} r \\
q^{\prime \prime} s^{\prime \prime}, & 0
\end{array}\right)
$$

Our main result is

Theorem 1. For the existence of a local isometry $f: T_{1} \rightarrow T_{2}$ the following conditions are necessary and sufficient:

(1) $\tau_{1}$ and $\tau_{2}$ are equivalent under $G L^{+}(2, Q)$;

(2a) If $T_{1}$ is nonample, then $r_{1} / r_{2}$ must be an integer;

(2b) If $T_{1}$ is ample, then $\left(r_{1}^{2} / r_{2}^{2}\right) a$ must be an integer $N$, and $N$ must be representable by the quadratic form

$$
\operatorname{det}\left(\kappa_{1} \widetilde{T}_{1}+\kappa_{2} \widetilde{T}_{2}\right)
$$

with suitable integers $\kappa_{1}$ and $\kappa_{2}$.

Proof. Since $f$ is a conformal covering we have necessarily (1) by (i). The following identity is readily verified:

$$
\frac{r_{1}^{2}}{r_{2}^{2}}|C|^{2} a= \begin{cases}\operatorname{det}\left(\kappa \widetilde{T}_{1}\right) & \text { for } T_{1} \text { nonample } \\ \operatorname{det}\left(\kappa_{1} \widetilde{T}_{1}+\kappa_{2} \widetilde{T}_{2}\right) & \text { for } T_{1} \text { ample }\end{cases}
$$

(The right hand side gives the number $N$ of sheets of the covering $f$ ). 
Together with the condition $|C|=1$ for local isometry it leads to (2a) and (2b). The sufficiency follows from (iii) and (iv).

In both cases we have the following consequences. A flat torus can cover a countably infinite set of tori by local isometries. For $T_{1}=$ $T_{2}$ a local isometry is a global isometry, since $|C|=1$ entails $N=1$. In general the existence of a local isometry $T_{1} \rightarrow T_{2}$ does not imply that there is also a local isometry $T_{2} \rightarrow T_{1}$; this occurs if and only if both $r_{1}=r_{2}$ and condition (1) are satisfied. (Then the tori still need not be globally isometric).

3. Homotopy classes. We show how the combination $\kappa_{1} \widetilde{T}_{1}+\kappa_{2} \widetilde{T}_{2}$ controls also the deformation properties of our maps. If the constant $D$ in (ii) is varied the map stays in the same homotopy class, but maps corresponding to different parameter values $\kappa$ or $\left(\kappa_{1}, \kappa_{2}\right)$ are not analytically homotopic (i.e., with analytic intermediately stages during the deformation), since the set of admissible values of $C$ is discrete. We show that they are not even homotopic in the ordinary sense.

Since the fundamental group $\pi_{1}(T)$ of a torus is Abelian the set $\mathscr{C}$ of homotopy classes of continuous maps $T_{1} \rightarrow T_{2}$ is in one-to-one correspondence with the set of all homomorphisms $\eta: \pi_{1}\left(T_{1}\right) \rightarrow \pi_{1}\left(T_{2}\right)$. Denoting by $L_{i}$ and $L_{i}^{\prime}(i=1,2)$ the path homotopy classes of two generating loops of $\pi_{1}\left(T_{i}\right)$, each such $\eta$ is characterized by the integral matrix

$$
\xi=\left(\begin{array}{ll}
\xi_{4}, & \xi_{3} \\
\xi_{2}, & \xi_{1}
\end{array}\right)
$$

given by

$$
\eta\left(L_{1}\right)=L_{2}^{\hat{\xi}_{1}} L_{2}^{\prime \hat{\tau}_{2}}, \eta\left(L_{1}^{\prime}\right)=L_{2}^{\hat{\xi}_{3}} L_{2}^{\prime \hat{\xi}_{4}} ;
$$

hence $\mathscr{C}$ is parametrized by $Z^{4}$. The subset $\left\{\xi \in Z^{4}: \operatorname{det} \xi \neq 0\right\}$ contains those points of $Z^{4}$ representing monomorphisms, hence it corresponds to the homotopy classes containing covering maps.

THEOREM 2. The subset of $Z^{4}$ corresponding to homotopy classes which contain analytic maps consists of

(a) 0 only if $\tau_{1}$ and $\tau_{2}$ are nonequivalent under $G L^{+}(2, Q)$;

(b) the 1-dimensional sublattice spanned by $\widetilde{T}_{1}$ if $\tau_{1}$ and $\tau_{2}$ are equivalent under $G L^{+}(2, Q)$ and both are nonample;

(c) the 2-dimensional sublattice spanned by $\widetilde{T}_{1}$ and $\widetilde{T}_{2}$ if $\tau_{1}$ and $\tau_{2}$ are equivalent under $G L^{+}(2, Q)$ and both are ample.

Proof. We prove only (c); (a) and (b) can be handled similarly. The generators $L_{i}, L_{i}^{\prime}$ of $\pi_{1}\left(T_{i}\right)$ are represented in $E_{i}$ by the segments $S_{i}, S_{i}^{\prime}$ joining the origin to $r_{i}$ and $r_{i} h_{i}$ respectively. The segments $S_{1}$ 
and $S_{1}^{\prime}$ are mapped by $F(z ; C, 0)$ (cf. (ii)) into segments from the origin of $E_{2}$ to the points

$$
\kappa_{1} r_{2}+\kappa_{2} s^{\prime \prime} q^{\prime \prime} r_{2} h_{2}
$$

and

$$
-\kappa_{2} r a^{\prime \prime} q^{\prime} r_{2}+\left(\kappa_{1} a+\kappa_{2} s^{\prime \prime} p a^{\prime}\right) r_{2} h_{2} \text {. }
$$

The former can be deformed into the two sides $\kappa_{1} r_{2}$ and $\kappa_{2} s^{\prime \prime} q^{\prime \prime} r_{2} h_{2}$ of a parallelogram parallel to $S_{2}$ and $S_{2}^{\prime}$. The first side represents $\kappa_{1}$ circuits of $L_{2}$, the second $\kappa_{2} s^{\prime \prime} q^{\prime \prime}$ contours of $L_{2}^{\prime}$. Similarly for $S_{1}^{\prime}$. Hence the homomorphism

$$
f_{*}: \pi_{1}\left(T_{1}\right) \longrightarrow \pi_{1}\left(T_{2}\right)
$$

induced by $f$ is determined by

$$
f_{*}\left(L_{1}\right)=L_{2}^{\kappa} L_{2}^{\prime \kappa_{2} s^{\prime \prime} q^{\prime \prime}}
$$

and

$$
f_{*}\left(L_{1}^{\prime}\right)=L_{2}^{-\kappa_{2} r a^{\prime \prime} q^{\prime}} L_{2}^{\prime \kappa_{1} a+\kappa_{2} s^{\prime \prime p a^{\prime}}} .
$$

This is equivalent to $\xi=\kappa_{1} \widetilde{T}_{1}+\kappa_{2} \widetilde{T}_{2}$.

The determination of all local isometries for two given tori is easy for the nonample case. In the ample case it involves the number of ways in which $N=\left(r_{1}^{2} / r_{2}^{2}\right) a$ can be represented by the quadratic form (4). Since this form is positive definite we have, in conjunction with Theorem 2:

Theorem 3. The number of homotopy classes of local isometries between two flat tori is finite.

We obtain an upper bound for this number as follows: From (3) we find

$$
\Re C=\frac{r_{2}}{r_{1}}\left(\kappa_{1}+\kappa_{2} s^{\prime \prime} \frac{p}{2 g^{\prime}}\right),
$$

which shows that $\Re C$ has the form $\left(r_{2} / r_{1}\right)\left(\gamma / 2 g^{\prime}\right)$, with $\gamma$ an integer. Substituting this in $|\Re C| \leqq|C|=1$ leads to

$$
|\gamma| \leqq 2 g^{\prime} \frac{r_{1}}{r_{2}} .
$$

From $(\mathfrak{I} C)^{2}=|C|^{2}-(\Re C)^{2}$ we deduce 
(6)

$$
\kappa_{2}^{2} q^{\prime \prime 2} s^{\prime \prime 2}\left(\mathfrak{T} h_{2}\right)^{2}=\frac{r_{1}^{2}}{r_{2}^{2}}-\frac{\gamma^{2}}{4 g^{\prime 2}}
$$

and

$$
\kappa_{1}=\frac{\gamma}{2 g^{\prime}}-\kappa_{2} s^{\prime \prime} \frac{p}{2 g^{\prime}} .
$$

Each of the $2\left[2 g^{\prime}\left(r_{1} / r_{2}\right)\right]+1$ integers $\gamma$ compatible with (5) leads to at most two pairs $\left(\kappa_{1}, \kappa_{2}\right)$ compatible with (6) and (7). Thus the number of homotopically different local isometries does not exceed $4\left[2 g^{\prime}\left(r_{1} / r_{2}\right)\right]+2$.

\section{BIBLIOGRAPHY}

1. H. Helfenstein, Analytic maps between tori, Bull. Amer. Math. Soc. Vol. 75, No. 4, 857-859.

2. J. A. Wolf, Spaces of constant curvature, New York, 1967.

Received July 9, 1969.

UNIVERSITY OF OTTAWA

OtTaWa, Canada 



\section{PACIFIC JOURNAL OF MATHEMATICS}

\section{EDITORS}

\author{
H. SAMELSON \\ Stanford University \\ Stanford, California 94305 \\ Richard Pierce \\ University of Washington \\ Seattle, Washington 98105
}

J. DUGUNDJI

Department of Mathematics

University of Southern California

Los Angeles, California 90007

BASIL GORDON*

University of California

Los Angeles, California 90024

\section{ASSOCIATE EDITORS}
E. F. BECKENBACH
B. H. NeUmanN
F. WOLF
K. YoSHIDA

\section{SUPPORTING INSTITUTIONS}

UNIVERSITY OF BRITISH COLUMBIA CALIFORNIA INSTITUTE OF TECHNOLOGY

UNIVERSITY OF CALIFORNIA MONTANA STATE UNIVERSITY

UNIVERSITY OF NEVADA

NEW MEXICO STATE UNIVERSITY

OREGON STATE UNIVERSITY

UNIVERSITY OF OREGON

OSAKA UNIVERSITY

UNIVERSITY OF SOUTHERN CALIFORNIA
STANFORD UNIVERSITY

UNIVERSITY OF TOKYO

UNIVERSITY OF UTAH

WASHINGTON STATE UNIVERSITY

UNIVERSITY OF WASHINGTON

AMERICAN MATHEMATICAL SOCIETY CHEVRON RESEARCH CORPORATION TRW SYSTEMS

NAVAL WEAPONS CENTER 


\section{Pacific Journal of Mathematics}

\section{Vol. 32, No. $1 \quad$ January, 1970}

Robert Alexander Adams, Compact Sobolev imbeddings for unbounded domains ........................................ 1

Bernhard Amberg, Groups with maximum conditions .................. 9

Tom M. (Mike) Apostol, Möbius functions of order k............... 21

Stefan Bergman, On an initial value problem in the theory of two-dimensional transonic flow patterns ................... 29

Geoffrey David Downs Creede, Concerning semi-stratifiable spaces ...... 47

Edmond Dale Dixon, Matric polynomials which are higher

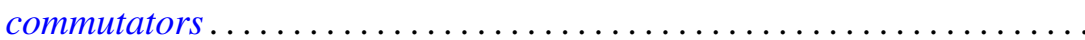

R. L. Duncan, Some continuity properties of the Schnirelmann density.

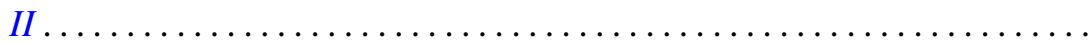

Peter Larkin Duren and Allen Lowell Shields, Coefficient multipliers of $H^{p}$

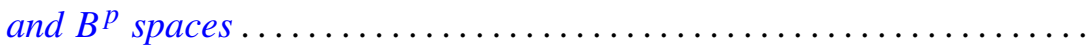

Hector O. Fattorini, On a class of differential equations for vector-valued

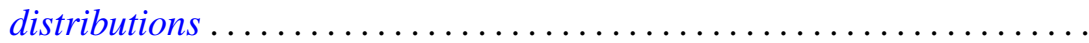

Charles Hallahan, Stability theorems for Lie algebras of derivations. . . . . . 105

Heinz Helfenstein, Local isometries of flat tori ................ 113

Gerald J. Janusz, Some remarks on Clifford's theorem and the Schur

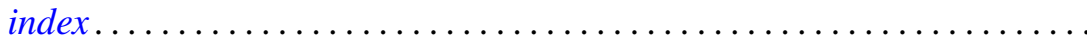

Joe W. Jenkins, Symmetry and nonsymmetry in the group algebras of discrete groups. ...

Herbert Frederick Kreimer, Jr., Outer Galois theory for separable

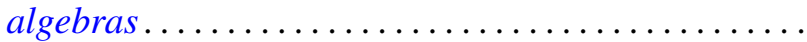

D. G. Larman and P. Mani, On visual hulls

R. Robert Laxton, On groups of linear recurrences. II. Elements of finite order.

Dong Hoon Lee, The adjoint group of Lie groups ...

James B. Lucke, Commutativity in locally compact rings

Charles Harris Scanlon, Rings of functions with certain Lipschitz

$$
\text { properties ............................... }
$$

Binyamin Schwarz, Totally positive differential systems .

James McLean Sloss, The bending of space curves into piecewise helical curves.

James D. Stafney, Analytic interpolation of certain multiplier spaces ...

Patrick Noble Stewart, Semi-simple radical classes.......

Hiroyuki Tachikawa, On left $\mathrm{QF}-3$ rings ...................... 255

Glenn Francis Webb, Product integral representation of time dependent nonlinear evolution equations in Banach spaces.... . . . 